\title{
PADRÕES MOTORES FUNDAMENTAIS DE CRIANÇAS DE UMA ESCOLA BAIANA
}

\author{
FUNDAMENTAL MOTOR PATTERNS OF CHILDREN OF A BAIAN SCHOOL
}

\author{
PATRONES FUNDAMENTALES DE MOTOR DE LOS NIÑOS DE UNA ESCUELA \\ BAIANA
}

\author{
Fábio Thomaz Melo ${ }^{1}$ \\ Kleonara Santos Oliveira ${ }^{2}$ \\ Angelo Maurício de Amorim ${ }^{3}$ \\ Claudio Bispo de Almeida ${ }^{4}$ \\ Ricardo Franklin de Freitas Mussi ${ }^{5}$
}

\begin{abstract}
Resumo
O objetivo deste estudo foi descrever a frequência dos padrões motores fundamentais de movimento de crianças matriculadas na educação infantil em escola de uma cidade do agreste baiano. As crianças foram submetidas a testes de avaliação motora. A amostra foi composta por 50 alunos da educação infantil, de ambos os sexos, 20 com quatro anos e $30 \mathrm{com}$ cinco anos de idade. As habilidades motoras fundamentais de pegar, chutar e saltar horizontalmente foram avaliadas em conformidade aos padrões de desenvolvimento esperados para cada idade. Os resultados apresentaram presença de atraso no desenvolvimento motor das crianças investigadas uma vez que os participantes deveriam estar no estágio elementar de desenvolvimento do movimento.
\end{abstract}

Palavras Chave: Padrões Motores; Desenvolvimento Motor; Educação Infantil.

\footnotetext{
Abstract

The aim of this study was to describe the frequency of the fundamental motor movement patterns of children enrolled in early childhood education at a school in a city in rural Bahia.

${ }^{1}$ Mestre em Ciências da Educação. Docente na Universidade do Estado da Bahia.

E-mail: fabiomello fsa@hotmail.com

ORCID: https://orcid.org/0000-0001-5106-7350

2 Mestranda em Ensino, Linguagem e Sociedade pela Universidade do Estado da Bahia. Docente na Universidade do Estado da Bahia.

E-mail: kleonara@yahoo.com.br

ORCID: https://orcid.org/0000-0003-1626-2294

3 Doutor em Educação Física. Docente na Universidade do Estado da Bahia.

E-mail: angeloamorim@gmail.com

ORCID: https://orcid.org/0000-0002-8477-7452

4 Doutorando em Enfermagem e Saúde pela Universidade Estadual do Sudoeste da Bahia. Mestre em Educação Física. Docente na Universidade do Estado da Bahia.

E-mail: claudio bispo de almeida@hotmail.com

ORCID: https://orcid.org/0000-0001-9486-7163

5 Doutor em Educação Física. Docente no Programa de Pós-graduação em Ensino, Linguagem e Sociedade da Universidade do Estado da Bahia.

E-mail: rimussi@yahoo.com.br

ORCID: http://orcid.org/0000-0003-1515-9121
} 
The children were submitted to motor assessment tests. The sample consisted of 50 early childhood education students, of both sexes, 20 with four years old and 30 with five years old. The fundamental motor skills of catching, kicking and jumping horizontally were assessed in accordance with the expected development patterns for each age. The results showed a delay in the motor development of the children investigated since the participants should be in the elementary stage of movement development.

Keywords: Motor patterns; Motor development; Child education.

\section{Resumen}

El objetivo de este estudio fue describir la frecuencia de los patrones fundamentales de movimiento motor de los niños matriculados en educación infantil en una escuela de una ciudad de la zona rural de Bahía. Los niños fueron sometidos a pruebas de evaluación motora. La muestra estuvo conformada por 50 estudiantes de educación infantil, de ambos sexos, 20 con cuatro años y 30 con cinco años. Se evaluaron las habilidades motoras fundamentales de agarrar, patear y saltar horizontalmente de acuerdo con los patrones de desarrollo esperados para cada edad. Los resultados mostraron un retraso en el desarrollo motor de los niños investigados, ya que los participantes deberían estar en la etapa elemental del desarrollo del movimiento.

Palavras clave: Patrones motores; Desarrollo motor; Educación Infantil.

\section{Introdução}

O desenvolvimento aplicado aos seres vivos é um processo sucessivo que tem seu início no nascimento e encerra-se na morte. O termo motor versa, sobretudo (pessoa, coisa) em movimento (GALLAHUE; OZMUN; GOODWAY, 2013). Então, o desenvolvimento motor (DM) resulta de alterações progressivas no comportamento motor, do simples para o complexo, ocorrida ao longo dos ciclos de vida humana.

O desenvolvimento infantil depende de diversos fatores, tais como, fatores metabólicos, motores, sociais e emocionais. A limitação em algum desses elementos acarreta dificuldade de aprendizagem na criança (WALLON, 1979). É importante ressaltar que o DM está atrelado a outras habilidades, uma vez que é da ação motriz que regula o aparecimento e o desenvolvimento das formações mentais, ou seja, o movimento é o pensamento em ato.

O DM é impactado pela estimulação e pelas variações ambientais (OLIVEIRA, 2015). O processo de maturação biológico estabelece limites quanto a capacidade física e a motricidade das crianças, mas o ritmo pode ser atrasado pela ausência de práticas ou antecipado por experiências que as incitem. 
Estímulos motores durante a educação infantil, apesar de reconhecida como uma fase curta, são importantes para o DM adequado, especialmente para que as crianças desenvolvam suas habilidades e tornem-se capazes de realizar as tarefas de maneira competente ao longo da vida. Fonseca, Beltrame e Tkac (2008, p.183) ressaltam que "o desenvolvimento motor de crianças pode estar relacionado a vários fatores, entre eles as variáveis do contexto de desenvolvimento no qual a criança participa ativamente".

Aspecto importante nas fases é que o movimento se desenvolve a partir da codificação das informações até a sua utilização permanente. Para que os alunos apresentem um desenvolvimento adequado são necessários dois fatores: experiências vivenciadas e maturação (MARQUES et al., 2013).

O modelo de ampulheta (Figura 1) proposto por Gallahue, Ozmun e Goodway (2013) ilustra a progressão entre as fases do DM, considerando faixas etárias e a expectativa dos estágios correspondentes.

FIGURA 1: Ampulheta triangulada ilustrativa do processo do desenvolvimento motor ao longo da vida

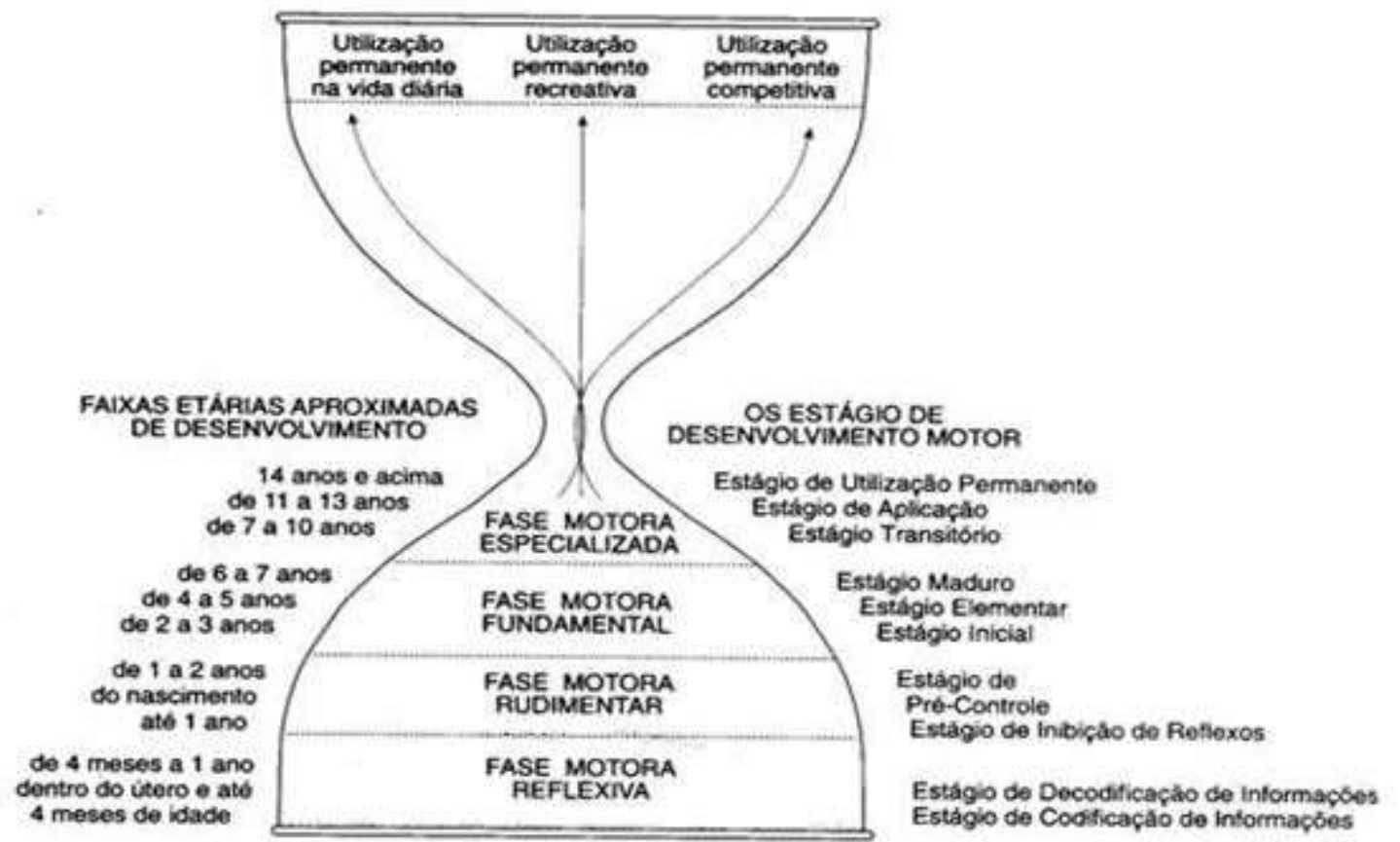

Fonte: Gallahue, Ozmun e Goodway (2013, p.69).

Dentre as fases do desenvolvimento infantil, a mais significativa é a referente as habilidades motoras fundamentais, pois promove alterações determinantes no futuro motor do sujeito. É considerada uma fase crítica e sensível, período 
em que o sujeito está vulnerável a determinada influência externa em certo período de tempo (LOPES; MAIA 2000).

Nesta fase e durante o seu processo de desenvolvimento, o indivíduo perpassa por três estágios: inicial, elementar e maduro (GALLAHUE, OZMUN E GOODWAY, 2013). Na fase inicial, a criança tenta executar um padrão de movimento fundamental, atingindo esse nível em torno dos dois a três anos; já na fase elementar, dependendo do processo maturacional, a criança tem maior coordenação e controle dos movimentos fundamentais, realçando entre quatro e cinco anos; e no estágio maduro, a criança tem eficiência na sua execução e coordenação mecânica, atingindo esse estágio próximo dos seis a sete anos.

Destarte, percebe-se a importância da criança desenvolver as habilidades motoras fundamentais na idade apropriada, o que possibilitará a plena exploração do ambiente que a rodeia.

Neste contexto, revisão sistemática identificou que a Educação Física escolar contribui para o desenvolvimento das habilidades motoras fundamentais nas crianças em idade escolar, demonstrando sua importância para o DM (FERNANDES et al., 2017). De maneira completar, análise bibliográfica destacou a relevância do professor de educação física para o entendimento de como a criança se movimenta, como ela aprende e como manifesta suas emoções e sentimentos, bem como o professor deve executar o trabalho de acordo com os estágios de DM (OLIVEIRA, 2002).

Também é essencial que o profissional conheça os níveis de DM das crianças para organizar ações motoras voltadas para as necessidades de diferentes grupos, a fim de possibilitar práticas mais efetivas, as quais estimulem as crianças a construir padrões de movimentos mais desenvolvidos para garantir a participação em atividades de movimentos por toda longevidade (VALENTINI, 2002).

Porém, no Brasil, pouco se tem estudado sobre a relação do DM acerca dos padrões básicos de movimento desde nascimento até a morte, o que acarreta consequências no processo de DM em indivíduos brasileiros (ISAYAMA; GALLARDO, 1998). Assim, acredita-se que essas questões bem delineadas tragam maiores conhecimentos aos docentes, refletindo positivamente, numa perspectiva de conhecer melhor o perfil de cada aluno a fim de possibilitar melhores oportunidades de desenvolvimento motriz. 
Mesmo diante da importância do adequado DM para potencializar o desenvolvimento infantil, ainda são restritas as análises que verificaram as habilidades/tarefas em escolares nas mais diversas realidades. Apesar dos impactos negativos do atraso motor para os escolares, ainda parece frágil o constructo informacional científico sobre a realidade do DM de crianças em diferentes realidades sócio-históricas. Então, o presente estudo objetiva descrever a frequência dos padrões motores fundamentais de movimento de crianças matriculadas na educação infantil em escola de uma cidade do agreste baiano.

\section{Métodos}

Esta análise trata-se de pesquisa descritiva, resultante da pesquisa: "avaliação dos padrões motores fundamentais de movimentos em crianças de 4 a 5 anos", autorizada pelo Comitê de Ética em Pesquisa da Faculdade de Tecnologias e Ciências (CEP/FTC), sob o CAAE: 91697180.00005032.

O desenvolvimento das atividades ocorreu em uma escola pública de educação infantil na cidade de Feira de Santana, Bahia, Brasil.

Como critérios de inclusão foram: ter entre quatro e cinco anos de idade, de ambos os sexos, ter iniciado a educação infantil no lócus da pesquisa e apresentação do termo de assentimento dos responsáveis. Já os critérios de exclusão foram: não apresentar deficiência e/ou desenvolvimento neuroatípico (conforme indicação de relatório médico presente na instituição), bem como não sentirem à vontade para participar das atividades propostas na pesquisa.

A população estudantil na faixa etária investigada totalizava 51 escolares matriculados na unidade escolar. A partir da aplicação dos critérios estabelecidos previamente, a amostra final foi composta por 50 escolares, 31 do sexo feminino e 19 do sexo masculino.

As crianças participaram dos testes de avaliação motora propostos por Gallahue, Ozmun e Goodway (2013). Após a filmagem das três tentavas foi realizada comparação as figuras preestabelecidas pela referência acerca dos estágios de padrões de movimentos sequenciais desenvolvimentais do chute, pegada e salto horizontal. A verificação do estágio de desenvolvimento em que as crianças 
estavam considerou como referência o movimento soberano, ou seja, a melhor execução das tentativas.

Para coleta de dados, foram selecionados seis avaliadores, sendo dois para cada habilidade motora fundamental. Durante as coletas foram utilizados os seguintes materiais: oito bolas de futsal penalty player 500 e a quadra poliesportiva, localizada na própria instituição. As filmagens foram realizadas em celular iphone-SE, versão 11.3.1 (15e302), com capacidade de $32 \mathrm{~GB}$, tela retina, tela LCD widescreen multi-touch com tecnologia IPS, e resolução de 1136 x 640 pixels, 326 ppp, câmera de 12 mp, abertura $f / 2.2$.

As turmas do Grupo 4 (crianças com quatro anos) e do Grupo 5 (escolares com cinco anos) foram divididas em três subgrupos, organizados em filas, e as atividades foram realizadas em estações. O subgrupo um realizava o salto horizontal, o subgrupo dois recebia a bola com as duas mãos e o subgrupo três chutava a bola com o pé dominante.

$\mathrm{Na}$ atividade do Salto, as crianças ultrapassavam uma linha tracejada a $10 \mathrm{~cm}$ do ponto de origem do salto, e na execução o salto deveria ser feito para frente (horizontalmente). Na atividade de recepção, um dos pesquisadores, a aproximadamente um metro do participante, jogava em trajetória retilínea a bola com as duas mãos na altura entre as regiões das cavidades torácicas para que fosse recebida com ambas as mãos. Na atividade do chute, deveriam chutar livremente uma bola de futsal colocada a sua frente para qualquer direção. Ressalta-se que as atividades não foram previamente demonstradas aos participantes.

A distribuição de frequências foi obtida por meio do Statisitical Package for the Social Sciences (IBM-SPSS), versão 22.

\section{Resultados e Discussão}

Entre os 50 escolares que participaram da pesquisa, identificou-se predominância do sexo feminino $(62,0 \%)$ e de crianças na faixa etária dos cinco anos, $60 \%$. Embora a localidade da escola seja em um bairro considerado não periférico, as crianças que ali estudam pertencem a comunidades periféricas. 
Especificamente quanto às fases do DM (Tabela 1) os avaliados apresentaram maiores participação da Fase Inicial na habilidade de pegada $(60,0 \%)$, de chute $(56,0 \%)$ e de salto horizontal $(62,0 \%)$.

Tabela 1. Fases de desenvolvimento motor conforme as habilidades em crianças. Feira de Santana, Bahia. 2018

\begin{tabular}{llll}
\hline & & \multicolumn{2}{c}{ Fases de desenvolvimento } \\
\hline Habilidade & Inicial & Elementar & Maduro \\
Pegada & $60,0 \%(30)$ & $36,0 \%(18)$ & $4,0 \%(2)$ \\
Chute & $56,0 \%(28)$ & $38,0 \%(19)$ & $6,0 \%(3)$ \\
Salto horizontal & $62,0 \%(31)$ & $34,0 \%(17)$ & $4,0 \%(2)$ \\
\hline
\end{tabular}

Fonte: dados da pesquisa.

A Tabela 1 demonstra que na habilidade pegada, dentre o total de participantes avaliados 60,0\% encontra-se no estágio inicial, 36,0\% elementar e apenas 4,0\% maduro, números estes que apresentam que a maiorias dos estudantes estão no estágio de DM abaixo do esperado quando confrontando com o apresentado por Gallahue, Ozmun e Goodway (2013).

A análise quanto ao desenvolvimento deste ou daquele domínio se justifica pela intenção do indivíduo e do contexto em que ele age (TANI et al., 1988). Então, não é justificável crítica do significado e da natureza da execução da criança dissociada de seu ambiente escolar de convivência (PERROTTI; MANOEL, 2001). O infante e o contexto escolar interagem mutuamente propiciando uma dinâmica desenvolvimentista.

Tabela 2. Fases de desenvolvimento motor na pegada conforme idade e sexo em crianças. Feira de Santana, Bahia. 2018.

\begin{tabular}{lccccc}
\hline \multirow{2}{*}{$\begin{array}{l}\text { Característica } \\
\text { demográfica }\end{array}$} & & \multicolumn{4}{c}{ Fases de desenvolvimento } \\
Idade & & & & & \\
& & & & & \\
& 4 anos & $26,0 \%(13)$ & $14,0 \%(7)$ & $0,0 \%(0)$ & $40,0 \%(20)$ \\
& 5 anos & $34,0 \%(17)$ & $22,0 \%(11)$ & $4,0 \%(2)$ & $60,0 \%(30)$ \\
Sexo & & $60,0 \%(30)$ & $36,0 \%(18)$ & $4,0 \%(2)$ & \\
& Feminino & $40,0 \%(20)$ & $22,0 \%(11)$ & $0,0 \%(0)$ & $62,0 \%(31)$ \\
& Masculino & $20,0 \%(10)$ & $14,0 \%(7)$ & $4,0 \%(2)$ & $38,0 \%(19)$ \\
& & $60,0 \%(30)$ & $36,0 \%(18)$ & $4,0 \%(2)$ & \\
\hline
\end{tabular}

Fonte: dados da pesquisa.

Em relação a idade (Tabela 2) $26,0 \%$ dos estudantes de quatro anos, dentre 20 participantes, estão no estágio inicial, $14 \%$ no estágio elementar e $0,0 \%$ 
no estágio maduro, confirmando que a maioria estão no estágio de DM abaixo do esperado, de acordo com Gallahue, Ozmun e Goodway (2013), se igualando aos achados do estudo de Marques (2013) o qual constatou-se que as crianças de quatro anos de idade se encontram em um nível abaixo do esperado. Já os de cinco anos, no total de 30 avaliados, $34 \%$ encontram-se no estágio inicial, $22,0 \%$ no estágio elementar e 4,0\% maduro, detectando que estes também estão no estágio de DM abaixo do esperado, quando comparado com o estabelecido por Gallahue, Ozmun e Goodway (2013), diferentes dos resultados de Marques (2013) e Paim (2003) os quais os avaliados da faixa etária encontravam-se dentro do estágio de DM proposto pelo autor.

Ao considerar o sexo das crianças, à Tabela 3 mostra que $40,0 \%$ dos escolares do sexo feminino (31 estudantes) encontram-se no estágio inicial, 22,0\% no estágio elementar e $0,0 \%$ no estágio maduro, apresentando que a maioria das meninas não estão no estágio de DM esperável quando conferido com o colocado por Gallahue, Ozmun e Goodway (2013), diferente dos resultados de Marques (2013) e Paim (2003) os quais os avaliados do sexo se concentravam a maioria no estágio elementar de DM proposto pelo autor Gallahue, Ozmun e Goodway (2013).

Já o sexo masculino (19 escolares) 20,0\% encontram-se no estágio inicial, $14,0 \%$ no elementar e $4,0 \%$ no maduro, também fortalecendo o discurso que a maioria dos meninos não estão no estágio de DM esperado quando defrontado com o proposto por Gallahue, Ozmun e Goodway (2013), se igualando aos achados de Marques (2013), o qual o sexo estudado, a maior porcentagem encontravam-se no estágio de DM inicial, e diferente do estudo de Paim (2003) o qual a sua maioria encontravam-se no estágio maduro de DM. 
Tabela 3. Fases de desenvolvimento motor no chute conforme idade e sexo em crianças. Feira de Santana, Bahia. 2018

\begin{tabular}{|c|c|c|c|c|c|}
\hline \multirow{2}{*}{$\begin{array}{l}\text { Característica } \\
\text { demográfica } \\
\text { ldade }\end{array}$} & \multicolumn{5}{|c|}{ Fases de desenvolvimento } \\
\hline & & Inicial & Elementar & Maduro & \\
\hline \multirow{2}{*}{ Sexo } & $\begin{array}{l}4 \text { anos } \\
5 \text { anos }\end{array}$ & $\begin{array}{l}28,0 \%(14) \\
28,0 \%(14) \\
56,0 \%(28)\end{array}$ & $\begin{array}{l}12,0 \%(6) \\
26,0 \%(13) \\
38,0 \%(19)\end{array}$ & $\begin{array}{c}0,0 \%(0) \\
6,0 \%(3) \\
6,0 \%(3)\end{array}$ & $\begin{array}{c}40 \%(20) \\
60,0 \%(30)\end{array}$ \\
\hline & $\begin{array}{l}\text { Feminino } \\
\text { Masculino }\end{array}$ & $\begin{array}{c}40,0 \%(20) \\
16,0 \%(8) \\
56,0 \%(28)\end{array}$ & $\begin{array}{c}22,0 \%(11) \\
16,0 \%(8) \\
38,0 \%(19)\end{array}$ & $\begin{array}{l}0,0 \%(0) \\
6,0 \%(3) \\
6,0 \%(3)\end{array}$ & $\begin{array}{l}62,0 \%(31) \\
38,0 \%(19)\end{array}$ \\
\hline
\end{tabular}

Fonte: dados da pesquisa.

Todos resultados terão como principal parâmetro de comparação, os autores Gallahue, Ozmun e Goodway (2013) por meio da ampulheta proposta por eles.

Quanto ao chute, a Tabela 3 indica que, do total analisados 56,0\% encontra-se no estágio inicial, 38,0\% elementar e apenas $6,0 \%$ maduro, tendo como resultado, o estágio de DM abaixo do esperado ao considerar a ampulheta comparativa (Gallahue, Ozmun e Goodway, 2013). Na Tabela 3, 28,0\% dos estudantes de quatro anos de idade, dentre 20 participantes, estão no estágio inicial, 12,0\% no estágio elementar e $0,0 \%$ no estágio maduro, enquanto os de 5 anos, no total de 30 avaliados, $28,0 \%$ encontram-se no estágio inicial, $26,0 \%$ no estágio elementar e $6,0 \%$ no maduro, não corroborando com a ampulheta proposta (GALLAHUE, OZMUN E GOODWAY, 2013), estando a maioria dos voluntários no estágio não esperado de DM.

A Tabela 4 aponta que 40,0\% dos escolares do sexo feminino (31 estudantes) encontram-se no estágio inicial, $22,0 \%$ no estágio elementar e $0,0 \%$ no estágio maduro. Discordante do previsto por Gallahue, Ozmun e Goodway (2013), a maior porção se concentra no nível abaixo esperado de DM. Já o sexo masculino (19 escolares) $16,0 \%$ encontra-se no estágio inicial, $16,0 \%$ no elementar e $6,0 \%$ no maduro. A maioria dos escolares não está no estágio de DM inadequado, porém a uma boa parcela se encontram. 
Tabela 4. Fases de desenvolvimento motor no salto horizontal conforme idade e sexo em crianças. Feira de Santana, Bahia. 2018

\begin{tabular}{|c|c|c|c|c|c|}
\hline \multirow{2}{*}{$\begin{array}{l}\text { Característica } \\
\text { demográfica } \\
\text { ldade }\end{array}$} & \multicolumn{5}{|c|}{ Fases de desenvolvimento } \\
\hline & & Inicial & elementar & Maduro & \\
\hline \multirow{7}{*}{ Sexo } & 4 anos & $28,0 \%(14)$ & $12,0 \%(6)$ & $0,0 \%(0)$ & $40,0 \%(20)$ \\
\hline & 5 anos & $34,0 \%(17)$ & $22,0 \%(11)$ & $4,0 \%(2)$ & $60,0 \%(30)$ \\
\hline & & $62,0 \%(31)$ & $34,0 \%(17)$ & $4,0 \%(2)$ & \\
\hline & & & & & \\
\hline & Feminino & $44,0 \%(22)$ & $18,0 \%(9)$ & $0,0 \%(0)$ & $62,0 \%(31)$ \\
\hline & Masculino & $18,0 \%(9)$ & $16,0 \%(8)$ & $4,0 \%(2)$ & $38,0 \%(19)$ \\
\hline & & $62,0 \%(31)$ & $34,0 \%(17)$ & $4,0 \%(2)$ & \\
\hline
\end{tabular}

Fonte: dados da pesquisa.

A Tabela 4 apresenta que, do total de crianças analisadas relativo ao salto horizontal, 62,0\% encontra-se no estágio inicial, 34,0\% elementar e apenas 4,0\% maduro. Este resultado não corrobora com o estudo de Marques (2013) e Paim (2003), os quais apontam que a maioria dos escolares avaliados se encontrava no estágio elementar de DM.

$\mathrm{Na}$ Tabela 4, 28,8\% dos estudantes de quatro anos, dentre 20 participantes, estão no estágio inicial, $12,0 \%$ no estágio elementar e $0,0 \%$ no estágio maduro, se igualando aos achados do estudo de Marques (2013) o qual constatou-se que as crianças de 4 anos se encontram em um nível abaixo do esperado de DM. Já os de cinco anos, dentre 30 participantes, 34,0\% encontram-se no estágio inicial, $22,0 \%$ no estágio elementar e 4,0\% maduro.

Ao se observar por sexo à Tabela 4 mostra que 44,0\% dos escolares do sexo feminino (31 estudantes) encontram-se no estágio inicial, 18,0\% no estágio elementar e $0,0 \%$ no estágio maduro. No sexo masculino (19 escolares) 18,0\% encontraram-se no estágio inicial, $16,0 \%$ no elementar e 4,0\% no maduro. De acordo com o presente estudo e comparando com o de Marques (2013) observou-se resultados diferentes, os quais na maioria dos alunos do sexo masculino se encontravam, na mesma proporção, nos estágios elementar e maduro do movimento locomotor saltar, enquanto o de Paim (2003) a sua concentração estava apenas no estágio elementar, divergente do presente estudo, que a sua maior concentração se encontra no estágio inicial.

Os resultados na Tabela 4 apontaram que há uma maior proporção dos alunos do sexo feminino no estágio inicial de desenvolvimento, diferentemente dos 
alunos estudados por Marques (2013) os quais metade das alunas estão no estágio inicial e a outra no estágio elementar. Enquanto no estudo de Paim (2003) a sua maior porcentagem encontravam-se apenas no estágio elementar. Deve-se considerar a existência de estudo que encontrou que o sexo não interfere nos padrões fundamentais de DM (chutar, saltar e rolar), mas encontrou diferenças na habilidade arremessar, em relação ao sexo masculino, tal estudo considerou as classes sociais em sua análise (PALMA; GUARÍGLIA; MARQUES, 2016).

De modo geral, na presente pesquisa, observou-se que as frequências dos estágios do DM foram abaixo do previsto. Espera-se que no período escolar as crianças estejam em estágios do DM condizentes com a sua idade (OLIVEIRA, 2002). Vale ressaltar que a prática de atividades psicomotoras pode melhorar as habilidades motoras contribuindo no DM de crianças, como foi observado em um estudo realizado com crianças de três a seis anos de idade da cidade de Monte Azul Paulista, no qual foram feitas intervenções e baterias de testes (RISSI; TEIXEIRA-ARROYO, 2018).

Neste sentido, observa-se que para facilitar a aprendizagem, devem ser considerados outros fatores, como por exemplo, uma relação de afeto entre professoraluno, sobretudo em cada fase da vida escolar e em suas diferentes etapas/estágios, os alunos podem ter momentos que sejam afetivamente marcantes em seu desempenho pedagógico (FARIAS; MAIA; OLIVEIRA, 2019).

Em um estudo sobre jogos e brincadeiras, nas aulas de educação física, foram considerados indispensáveis, sobretudo por conta do envolvimento emocional proporcionado por essas atividades motoras, contribuindo na formação humana das crianças pesquisadas (MAIA; FARIAS; OLIVEIRA, 2020). Tem-se, assim, que a ludicidade pode permitir que criança explore melhor o seu potencial (FARIAS; MAIA; OLIVEIRA, 2019).

Toda análise deve considerar algumas limitações inerentes. Neste sentido, o presente estudo apresenta a impossibilidade de generalização dos resultados, por conta de seu desenho amostral. Entretanto, pode-se destacar como vantagem da presente pesquisa, o conhecimento sobre a predominância das fases de DM nos escolares participantes, o que pode contribuir para a elaboração e/ou aperfeiçoamento de programas que estimulem e/ou ampliem o desenvolvimento das habilidades deste grupo de criança 


\section{Conclusão}

A partir do contexto acima, pode-se concluir que, em todas as sequências avaliadas a maioria do avaliados apresenta resultado não condizente com o preconizado na ampulheta elaborada por Gallahue, Ozmun e Goodway, que aponta que nas idades avaliadas todos deveriam estar no estágio elementar do movimento, entretanto, as baixas frequências predominaram no DM como um todo.

Com base nos resultados do presente estudo, reforça-se a importância da necessidade de aulas de educação física, com profissionais habilitados, tendo em vista que muitas escolas não exploram essas aulas de forma adequada.

\section{REFERÊNCIAS}

FARIAS, A. L. P.; MAIA, D. F.; OLIVEIRA, M. A. T. Lúdico e a afetividade no processo ensino aprendizagem. Cenas Educacionais, v.2, n.2, p.25-41, 2019.

FERNANDES, L.B.G. et al. O contributo da Educação Física para o desenvolvimento motor: uma revisão sistemática. Gymnasium, v.2, n.2, p.1-6, 2017.

FONSECA, F. R.; BELTRAME, T. S.; TKAC, C. M. Relação entre o Nível de Desenvolvimento Motor e Variáveis do Contexto de Desenvolvimento de Crianças. Revista da Educação Física/UEM, v.19, n.2, p.183-194, 2008.

\section{GALLAHUE, D. L.; OZMUN, J. C.; GOODWAY, J. D. Compreendendo o} Desenvolvimento Motor: bebês, crianças, adolescentes e adultos. 7. ed. Porto Alegre: ARTMED, 2013. 487p.

ISAYAMA, H. F.; GALLARDO, J. S. P. Desenvolvimento Motor: Análise dos Estudos Brasileiros sobre Habilidades Motoras Fundamentais. Revista da Educação Física/UEM, v.9 n.1, p.75-82, 1998.

LOPES, V. P.; MAIA, J. A. R. Períodos críticos ou sensíveis: revisitar um tema polêmico à luz da investigação empírica. Revista Paulista de Educação Física, v.14, n.2, p.40-128, 2000.

MAIA, D. F.; FARIAS, A. L. P.; OLIVEIRA, M. A. T. Jogos e brincadeiras nas aulas de educação física para o desenvolvimento da criança. Cenas Educacionais, v. 3, p.e8623, 2020. 
MARQUES, et al. Desenvolvimento motor: padrões motores fundamentais de movimento em crianças de 4 e 5 anos de idade. Lecturas Educación Física y Deportes, v.18, n.186, 2013.

OLIVEIRA, G. C. Psicomotricidade e reeducação num enfoque psicopedagógico. 20. Ed. Petrópolis, RJ: Vozes, 2015.

OLIVEIRA, J. A. Padrões motores fundamentais: implicações e aplicações na educação física infantil. Interação, v.6, n.6, p.37-42, 2002.

PAIM, M. C. C. Desenvolvimento motor de crianças pré-escolares entre 5 e 6 anos. Lecturas: Educación Física y Deportes, v.8, n.58, 2003.

PALMA, L.; GUARÍGLIA, D. A.; MARQUES, I. Análise do desenvolvimento motor de crianças em diferentes classes sociais. Coleção Pesquisa em Educação Física, v. 15, n. 2, p. 35-43, 2016.

PERROTTI, A. C.; MANOEL, E. J. Uma visão epigenética do desenvolvimento motor. Revista. Brasileira de. Ciência e Movimento, v.9, n.4, p.77-82, 2001.

RISSI, J. B. D.; TEIXEIRA-ARROYO, C. Efeito de um circuito de atividades psicomotoras no desenvolvimento motor de crianças de 3 a 6 anos. Educação Física UNIFAFIBE, v.6, n.1, p.43-55, 2018.

TANI, G. et al. Educação física escolar: fundamentos de uma abordagem desenvolvimentista. São Paulo, Editora da Universidade de São Paulo, 1988.

VALENTINI, N. C. Percepções de competências de desenvolvimento motor de meninos e meninas: um estudo transversal. Movimento, v.8, n.2, p.51-62, 2002.

WALLON, H. Do ato do pensamento - Ensaio de psicologia comparada. Lisboa: Moraes, 1979.

Manuscrito recebido em: 28 de novembro de 2020

Aprovado em: 29 de dezembro de 2020

Publicado em: 29 de dezembro de 2020 